\title{
Reflexivity and approximate fixed points
}

\author{
by \\ Eva Matoušková (Praha) and Simeon Reich (Haifa)
}

\begin{abstract}
A Banach space $X$ is reflexive if and only if every bounded sequence $\left\{x_{n}\right\}$ in $X$ contains a norm attaining subsequence. This means that it contains a subsequence $\left\{x_{n_{k}}\right\}$ for which $\sup _{f \in S_{X^{*}}} \lim \sup _{k \rightarrow \infty} f\left(x_{n_{k}}\right)$ is attained at some $f$ in the dual unit sphere $S_{X^{*}}$. A Banach space $X$ is not reflexive if and only if it contains a normalized sequence $\left\{x_{n}\right\}$ with the property that for every $f \in S_{X^{*}}$, there exists $g \in S_{X^{*}}$ such that $\limsup _{n \rightarrow \infty} f\left(x_{n}\right)<\liminf _{n \rightarrow \infty} g\left(x_{n}\right)$. Combining this with a result of Shafrir, we conclude that every infinite-dimensional Banach space contains an unbounded closed convex set which has the approximate fixed point property for nonexpansive mappings.
\end{abstract}

1. Introduction. Let $M$ be a complete metric space and let $T: M \rightarrow M$ be a strict contraction, that is, let $T$ be Lipschitz with a constant less than one. By a classical theorem of Banach, $T$ has a fixed point $x=T(x)$. For contractions this need not be so. For example, it is readily checked that

$$
C=\{x \in C[0,1]: 0 \leq x \leq 1, x(0)=0, x(1)=1\}
$$

is a closed, convex and bounded subset of the space $C[0,1]$ of all real continuous functions on $[0,1]$ and that $T: C \rightarrow C$ defined by

$$
T x(t)=t x(t)
$$

satisfies $\|T(x)-T(y)\|<\|x-y\|$ for $x \neq y$ in $C$. However, $T$ has no fixed points. It does have, though, approximate fixed points. If $x_{n}$ is the $n$th power $x_{n}(t)=t^{n}$, then $\left\|x_{n}-T\left(x_{n}\right)\right\| \rightarrow 0$. This turns out to be the case in general, even for nonexpansive mappings.

Let $C$ be a closed convex subset of a Banach space $X$. Recall that a mapping $T: C \rightarrow C$ is called nonexpansive if $\|T x-T y\| \leq\|x-y\|$ for all

2000 Mathematics Subject Classification: Primary 46B10, 47H10; Secondary 47H09.

Key words and phrases: approximate fixed point property, convex set, norm attaining sequence, reflexive Banach space.

The first author was partially supported by the Czech Academy of Sciences grant GAAV-A1019103 and by Kepler University in Linz, Austria. The second author was partially supported by the Israel Science Foundation founded by the Israel Academy of Sciences and Humanities (grant 592/00) and by the Fund for the Promotion of Research at the Technion. 
$x, y \in C$. The subset $C$ is said to have the approximate fixed point property (AFPP) for nonexpansive mappings if $\inf \{\|x-T x\|: x \in C\}=0$ for all nonexpansive self-mappings of $C$. It is easy to see that every closed convex and bounded $C$ has this property. However, there are also unbounded $C$ with this property. For instance, it is shown in [R2] that a closed convex subset of a reflexive Banach space has the AFPP if and only if it does not contain any half-line. For example, $\left\{\left(x_{1}, x_{2}, \ldots\right) \in \ell_{2}:\left|x_{n}\right| \leq n\right\}$ is such an unbounded closed convex set. In [Sh], Shafrir shows that the "half-line test" works exactly in reflexive spaces. That is, a Banach space $X$ is reflexive if and only if every closed convex $C \subset X$ which does not contain any half-lines has the AFPP. In the same paper, Shafrir also characterizes those closed convex subsets of a Banach space (or, more generally, a complete hyperbolic metric space) which have the AFPP as those which, roughly speaking, do not contain any "approximate metric half-line". Using this characterization, he proves that every infinite-dimensional Banach space which does not contain an isomorphic copy of $\ell_{1}$ contains an unbounded closed convex subset with the AFPP. He also constructs such a subset in $\ell_{1}$. However, he has left open the question whether every infinite-dimensional Banach space contains such a subset. (Note that if $X$ is finite-dimensional and a convex $C \subset X$ does not contain any half-lines, then $C$ is bounded. Hence in this case either $C$ is bounded and has the fixed point property for nonexpansive mappings, or it is unbounded and does not even have the AFPP.)

In Corollary 4.4, we answer Shafrir's question in the affirmative. This is done by providing a characterization of reflexive Banach spaces in Theorem 4.3. We show there that a Banach space is reflexive if and only if every bounded sequence $\left\{x_{n}\right\}$ in $X$ contains a norm attaining subsequence. That is, it contains a subsequence $\left\{x_{n_{k}}\right\}$ for which $\sup _{S_{X^{*}}} \lim \sup _{k \rightarrow \infty} f\left(x_{n_{k}}\right)$ is attained at some $f$ from the dual unit sphere $S_{X^{*}}$. Also, a Banach space $X$ is not reflexive if and only if it contains a normalized sequence $\left\{x_{n}\right\}$ with the property that for every $f \in S_{X^{*}}$, there exists $g \in S_{X^{*}}$ such that $\limsup _{n \rightarrow \infty} f\left(x_{n}\right)<\liminf _{n \rightarrow \infty} g\left(x_{n}\right)$. In [Sh] Shafrir calls sequences with this property $(\mathrm{P})$-sequences. He proves that if $\left\{x_{n}\right\}$ is a $(\mathrm{P})$-sequence, then $\overline{\operatorname{conv}}\left\{n x_{n}: n \in \mathbb{N}\right\}$ has the AFPP.

When characterizing reflexive Banach spaces, we make use of the proof of the nonseparable case of James's theorem as presented in [S]. For more information on the approximate fixed point property for nonexpansive mappings see, for example, $[\mathrm{GR}],[\mathrm{GK}],[\mathrm{EK}],[\mathrm{K}]$ and the references mentioned therein.

By $S_{X}$ we denote the unit sphere of a Banach space $X$; by $X^{*}$ we denote the dual of $X$. By $\zeta, \eta, \ldots$ we denote subsequences of the sequence of natural numbers. 
2. Preliminaries. The following definition appears in $[\mathrm{Sh}]$.

Definition 2.1. Let $X$ be a Banach space. A sequence $\left\{x_{n}\right\}_{n=1}^{\infty} \subset S_{X}$ is called a $(\mathrm{P})$-sequence if for every $f \in S_{X^{*}}$, there exists $g \in S_{X^{*}}$ such that $\limsup _{n \rightarrow \infty} f\left(x_{n}\right)<\liminf _{n \rightarrow \infty} g\left(x_{n}\right)$.

Clearly, a subsequence of a $(\mathrm{P})$-sequence is again a $(\mathrm{P})$-sequence. The following is a straightforward consequence of the Hahn-Banach theorem.

Lemma 2.2. Suppose $Y$ is a closed subspace of a Banach space $X$ and $\left\{x_{n}\right\}_{n=1}^{\infty} \subset S_{Y}$ is a $(\mathrm{P})$-sequence in $Y$. Then $\left\{x_{n}\right\}_{n=1}^{\infty}$ is a $(\mathrm{P})$-sequence in $X$ as well.

Proof. Let $f \in S_{X^{*}}$ be given. Denote by $a$ the norm of $f$ when restricted to $Y$. Clearly, $a \leq 1$. If $a=0$, we choose any $g \in S_{Y^{*}}$ with $\liminf _{n \rightarrow \infty} g\left(x_{n}\right)>0$; the existence of such a functional $g$ follows directly from the definition of a $(\mathrm{P})$-sequence. If $0<a \leq 1$, we choose $g \in S_{Y^{*}}$ so that $\lim _{\sup _{n \rightarrow \infty}} f\left(x_{n}\right) / a<\liminf _{n \rightarrow \infty} g\left(x_{n}\right)$. In both cases we use the Hahn-Banach theorem to extend $g$ to a norm-one functional on $X$.

Quite often, we will make use of the following description of $(\mathrm{P})$-sequences. Note that a sequence $\left\{x_{n}\right\}$ satisfying (1) is called a Pryce sequence on p. 264 of [Sch].

Lemma 2.3. Let $X$ be a Banach space and let $\left\{x_{n}\right\} \subset S_{X}$ satisfy

$$
\sup _{f \in S_{X^{*}}} \limsup _{n \rightarrow \infty} f\left(x_{n}\right)=\sup _{f \in S_{X^{*}}} \liminf _{n \rightarrow \infty} f\left(x_{n}\right) .
$$

If the supremum on the left-hand side is not attained, then $\left\{x_{n}\right\}$ is a $(\mathrm{P})$ sequence. Conversely, if $\left\{x_{n}\right\}$ is a $(\mathrm{P})$-sequence, then (1) is satisfied and neither of the suprema is attained.

Proof. Suppose (1) is satisfied and the supremum on the left-hand side is not attained. Let $f \in S_{X^{*}}$. Then

$$
\limsup _{n \rightarrow \infty} f\left(x_{n}\right)<\sup _{h \in S_{X^{*}}} \limsup _{n \rightarrow \infty} h\left(x_{n}\right)=\sup _{h \in S_{X^{*}}} \liminf _{n \rightarrow \infty} h\left(x_{n}\right) .
$$

Therefore there exists a functional $g \in S_{X^{*}}$ such that $\lim _{\sup _{n \rightarrow \infty}} f\left(x_{n}\right)<$ $\liminf _{n \rightarrow \infty} g\left(x_{n}\right)$.

For the converse, observe that, trivially, for any bounded sequence $\left\{x_{n}\right\}$ and $f \in X^{*}$,

$$
\limsup _{n \rightarrow \infty} f\left(x_{n}\right) \geq \liminf _{n \rightarrow \infty} f\left(x_{n}\right) .
$$

Hence $\sup _{f \in S_{X^{*}}} \lim \sup _{n \rightarrow \infty} f\left(x_{n}\right) \geq \sup _{f \in S_{X^{*}}} \liminf _{n \rightarrow \infty} f\left(x_{n}\right)$. The definition of a (P)-sequence provides the other inequality needed for (1). By (1) and (2), if $\sup _{h \in S_{X^{*}}} \liminf _{n \rightarrow \infty} h\left(x_{n}\right)$ is attained at some $f \in S_{X^{*}}$, then 
$\sup _{h \in S_{X^{*}}} \lim \sup _{n \rightarrow \infty} h\left(x_{n}\right)$ is attained at this $f$ as well. But for this particular $f$, this fact contradicts the existence of the functional $g$ from the definition of a $(\mathrm{P})$-sequence.

If $X$ is a Banach space and $x \in X$, then $x$ attains its norm on the unit ball of $X^{*}$. If every $f \in X^{*}$ attains its norm on the unit ball of $X$, then $X$ is, according to the James theorem $[\mathrm{J}]$, reflexive. In Theorem 4.3 we establish a parallel to this result. Suppose that every bounded sequence in $X$ contains a subsequence which "attains its norm" on the unit ball of $X^{*}$. Then $X$ is reflexive.

Definition 2.4. Let $X$ be a Banach space. We call a bounded sequence $\left\{x_{n}\right\}_{n=1}^{\infty}$ in $X$ norm attaining if $\sup _{f \in S_{X^{*}}} \limsup _{n \rightarrow \infty} f\left(x_{n}\right)$ is attained on $S_{X^{*}}$.

The following lemma shows that $(\mathrm{P})$-sequences and sequences which do not attain their norm are closely related.

Lemma 2.5. Let $X$ be a Banach space and let $\left\{x_{n}\right\} \subset S_{X}$. If $\left\{x_{n}\right\}$ is $a(\mathrm{P})$-sequence, then no subsequence thereof is norm attaining. Conversely, if $\left\{x_{n}\right\}$ contains no norm attaining subsequences, then it contains a $(\mathrm{P})$ sequence.

Consequently, a Banach space $X$ contains no $(\mathrm{P})$-sequences if and only if every bounded sequence in $X$ contains a norm attaining subsequence.

Proof. Every subsequence of a $(\mathrm{P})$-sequence is also a $(\mathrm{P})$-sequence, so it is not norm attaining by Lemma 2.3 .

Suppose the sequence $\left\{x_{n}\right\}$ contains no norm attaining subsequences. By Lemma 3.1 below, it contains a subsequence $\left\{x_{n_{k}}\right\}$ such that

$$
\sup _{f \in S_{X^{*}}} \limsup _{k \rightarrow \infty} f\left(x_{n_{k}}\right)=\sup _{f \in S_{X^{*}}} \liminf _{k \rightarrow \infty} f\left(x_{n_{k}}\right) .
$$

By Lemma 2.3, $\left\{x_{n_{k}}\right\}$ is a $(\mathrm{P})$-sequence.

The last statement of the lemma for norm-one sequences is just a reformulation of the previous two. Hence, to finish the proof it is enough to observe that if there is a bounded sequence $\left\{x_{n}\right\}$ with no norm attaining subsequences, then there is a normalized sequence which has this property as well. Clearly, $\left\{x_{n}\right\}$ contains a subsequence $\left\{x_{n_{k}}\right\}$ with $\lim _{k \rightarrow \infty}\left\|x_{n_{k}}\right\|=a>0$. Then $\limsup _{k \rightarrow \infty} f\left(x_{n_{k}} /\left\|x_{n_{k}}\right\|\right)=\limsup _{k \rightarrow \infty} f\left(x_{n_{k}}\right) / a$ for all $f \in X^{*}$. Hence, if the supremum $\sup _{S_{X^{*}}} \lim \sup _{k \rightarrow \infty} f\left(x_{n_{k}}\right)$ is not attained, then $\sup _{S_{X^{*}}} \lim \sup _{k \rightarrow \infty} f\left(x_{n_{k}} /\left\|x_{n_{k}}\right\|\right)$ is not attained either.

3. Tools from the proof of the James theorem. Recall that according to the James theorem $[\mathrm{J}]$, a Banach space $X$ is reflexive if and only if every $f \in X^{*}$ attains its norm. In this section we reproduce two lemmata 
which can be used to prove this theorem. For the convenience of the reader, we also provide proofs.

The first lemma is taken from $[\mathrm{P}]$. The following trivial observation might help to understand its statement and the proof. Suppose $\left\{x_{n}\right\}$ is a bounded sequence in $\ell_{\infty}(S) \operatorname{such}$ that $\sup _{S} \lim \sup x_{n}$ is attained at some $s \in S$. Choose a subsequence $\zeta$ of $\mathbb{N}$ so that $\lim x_{\zeta(n)}(s)=\lim \sup x_{n}(s)$. Then for any subsequence $\eta$ of $\zeta$ we have $\sup _{S} \lim \sup x_{\zeta(n)}=\sup _{S} \lim \sup x_{\eta(n)}$ and $\sup _{S} \lim \sup x_{\zeta(n)}=\sup _{S} \liminf x_{\zeta(n)}$. In the lemma, the existence of points where either the supremum or the infimum are attained is not assumed and the equalities are required at separably many translates. In the proof, the resulting difficulty is overcome by diagonalization arguments.

Lemma 3.1. Let $S$ be a set, $\left\{x_{n}\right\}$ a bounded sequence in $\ell_{\infty}(S)$, and let $X$ be a separable subset of $\ell_{\infty}(S)$. Then

(i) there exists a subsequence $\zeta$ of $\mathbb{N}$ such that for every subsequence $\eta$ of $\zeta$ and every $x \in X$, we have

$$
\begin{aligned}
& \sup _{s \in S} \limsup _{n \rightarrow \infty}\left(x-x_{\zeta(n)}\right)(s)=\sup _{s \in S} \limsup _{n \rightarrow \infty}\left(x-x_{\eta(n)}\right)(s), \\
& \sup _{s \in S} \liminf _{n \rightarrow \infty}\left(x-x_{\zeta(n)}\right)(s)=\sup _{s \in S} \liminf _{n \rightarrow \infty}\left(x-x_{\eta(n)}\right)(s) ;
\end{aligned}
$$

(ii) if $\zeta$ is a sequence as in (i), then for all $x \in X$, we have

$$
\begin{aligned}
& \inf _{s \in S} \limsup _{n \rightarrow \infty}\left(x-x_{\zeta(n)}\right)(s)=\inf _{s \in S} \liminf _{n \rightarrow \infty}\left(x-x_{\zeta(n)}\right)(s), \\
& \sup \limsup _{s \in S}\left(x-x_{\zeta(n)}\right)(s)=\sup _{s \in S} \liminf _{n \rightarrow \infty}\left(x-x_{\zeta(n)}\right)(s) .
\end{aligned}
$$

Proof. (i) We show that it is possible to ensure the first equality of (i); to ensure the second one as well, one repeats the argument in a similar fashion.

First, we show that for a fixed $x \in X$, there is a subsequence $\zeta$ satisfying the first equality of (i). We fix some sequence $\left\{\varepsilon_{i}\right\}, \varepsilon_{i}>0$, with $\lim \varepsilon_{i}=0$. We choose inductively sequences $\mathbb{N}=\zeta_{0} \supset \zeta_{1} \supset \ldots$ and $s_{i} \in S$ so that there exists

$$
\lim _{n \rightarrow \infty}\left(x-x_{\zeta_{i+1}(n)}\right)\left(s_{i+1}\right) \geq \sup \lim \sup \left(x-x_{\zeta_{i}}\right)-\varepsilon_{i+1} .
$$

Let $\zeta(n)=\zeta_{n}(n)$ be the diagonal of $\left\{\zeta_{i}\right\}$. Let $\eta$ be a subsequence of $\zeta$. Then for any $i \in \mathbb{N}$, we have

$$
\begin{aligned}
\sup \lim \sup \left(x-x_{\eta}\right) & \leq \sup \lim \sup \left(x-x_{\zeta}\right) \leq \sup \lim \sup \left(x-x_{\zeta_{i}}\right) \\
& \leq \lim \left(x-x_{\zeta_{i+1}}\right)\left(s_{i+1}\right)+\varepsilon_{i+1} \\
& =\lim \left(x-x_{\eta}\right)\left(s_{i+1}\right)+\varepsilon_{i+1} \\
& \leq \sup \lim \sup \left(x-x_{\eta}\right)+\varepsilon_{i+1}
\end{aligned}
$$

The first inequality follows from $\eta \subset \zeta$, while the second follows from the fact that except for the first $i$ members, $\zeta \subset \zeta_{i}$; similarly, the equality follows 
from the fact that except for the first $i+1$ members, $\eta \subset \zeta_{i+1}$. Consequently, $\sup \lim \sup \left(x-x_{\eta}\right)=\sup \lim \sup \left(x-x_{\zeta}\right)$.

Another diagonalization allows us to choose $\zeta$ which will work for a dense countable subset $\left\{y_{j}\right\}$ of $X$. Such a $\zeta$ then works for all $x \in X$.

We now choose inductively sequences $\zeta_{1} \supset \zeta_{2} \supset \ldots$ so that for all subsequences $\eta$ of $\zeta_{j}, \sup \lim \sup \left(y_{j}-x_{\zeta_{j}}\right)=\sup \lim \sup \left(y_{j}-x_{\eta}\right)$. Let $\zeta(n)=\zeta_{n}(n)$ be the diagonal of $\left\{\zeta_{j}\right\}$. If $j \in \mathbb{N}$ and $\eta$ is a subsequence of $\zeta$, then

$$
\begin{aligned}
\sup \lim \sup \left(y_{j}-x_{\eta}\right) & \leq \sup \lim \sup \left(y_{j}-x_{\zeta}\right)=\sup \lim \sup \left(y_{j}-x_{\zeta_{j}}\right) \\
& =\sup \lim \sup \left(y_{j}-x_{\eta}\right) .
\end{aligned}
$$

Here the inequality holds because $\eta \subset \zeta$, the first equality holds since except for the first $j$ members, $\zeta \subset \zeta_{j}$, and similarly, the last equality holds because except for the first $j$ members, $\eta \subset \zeta_{j}$.

(ii) We show that the second equality of (i) implies the second equality of (ii). That the first equality of (i) implies the first equality of (ii) can be proved similarly.

Let $\zeta$ be as in (i) and let $\varepsilon>0$. Choose $s \in S$ and a subsequence $\eta$ of $\zeta$ so that there exists

$$
\lim \left(x-x_{\eta}\right)(s) \geq \sup \lim \sup \left(x-x_{\zeta}\right)-\varepsilon .
$$

Then for $x \in X$,

$$
\begin{aligned}
\sup \lim \sup \left(x-x_{\zeta}\right) & \geq \sup \liminf \left(x-x_{\zeta}\right)=\sup \liminf \left(x-x_{\eta}\right) \\
& \geq \lim \left(x-x_{\eta}\right)(s) \geq \sup \lim \sup \left(x-x_{\zeta}\right)-\varepsilon ;
\end{aligned}
$$

the equality above is a consequence of the second equality of (i). As $\varepsilon>0$ was arbitrary, we conclude that $\sup \lim \sup \left(x-x_{\zeta}\right)=\sup \liminf \left(x-x_{\zeta}\right)$.

The following technical lemma appears in [S]. It can be used, for example, to prove the inequality presented by Simons in [Si]. It should be understood as an upper estimate of a certain smallest supremum. In the proofs it is used, roughly speaking, as follows. Assume that $y=(1-\varepsilon) \sum_{k=1}^{\infty} \varepsilon^{k-1} y_{k}$ attains its supremum on $S$. Then $\alpha_{1}$ is, up to a small error, bounded from above by $\sup _{S} \lim \sup _{n \rightarrow \infty} z_{n}$.

LEMma 3.2. Let $S$ be a set and let $\left\{z_{n}\right\}$ be a bounded sequence in $\ell_{\infty}(S)$. Let $0<\delta, \varepsilon<1$, and let $A_{n}=\operatorname{conv}\left\{z_{i}: i \geq n\right\}$. Choose $y_{1} \in A_{1}$ such that

$$
\alpha_{1}=\sup _{S} y_{1} \leq \inf _{y \in A_{1}} \sup _{S} y+\delta(\varepsilon / 2)
$$

and $y_{m+1} \in A_{m+1}$ such that

$$
\alpha_{m+1}=\sup _{S}\left(\sum_{k=1}^{m+1} \varepsilon^{k-1} y_{k}\right) \leq \inf _{y \in A_{m+1}} \sup _{S}\left(\sum_{k=1}^{m} \varepsilon^{k-1} y_{k}+\varepsilon^{m} y\right)+\delta(\varepsilon / 2)^{m+1} .
$$


Then for each $m \in \mathbb{N}$ we have

$$
\lim _{n \rightarrow \infty} \alpha_{n} \geq \alpha_{m}+\frac{\varepsilon^{m}}{1-\varepsilon}\left(\alpha_{1}-(1+\varepsilon) \delta\right) .
$$

Proof. Set $\alpha_{0}=0$. Since $\left(y_{m}+\varepsilon y_{m+1}\right) /(1+\varepsilon) \in A_{m}$, the definition of $\alpha_{m}$ implies that

$$
\begin{aligned}
(1+\varepsilon) \alpha_{m} \leq & \sup \left[(1+\varepsilon) \sum_{k=1}^{m-1} \varepsilon^{k-1} y_{k}+\varepsilon^{m-1} y_{m}+\varepsilon^{m} y_{m+1}\right] \\
& +\delta(1+\varepsilon)(\varepsilon / 2)^{m} \\
\leq & \sup \left(\sum_{k=1}^{m+1} \varepsilon^{k-1} y_{k}\right)+\varepsilon \sup \left(\sum_{k=1}^{m-1} \varepsilon^{k-1} y_{k}\right)+\delta(\varepsilon / 2)^{m}(1+\varepsilon) \\
= & \alpha_{m+1}+\varepsilon \alpha_{m-1}+\delta(\varepsilon / 2)^{m}(1+\varepsilon) .
\end{aligned}
$$

Consequently,

$$
\alpha_{m+1}-\alpha_{m} \geq \varepsilon\left(\alpha_{m}-\alpha_{m-1}\right)-\delta(\varepsilon / 2)^{m}(1+\varepsilon) .
$$

By iterating this inequality we obtain

$$
\begin{aligned}
\alpha_{m+1}-\alpha_{m} & \geq \varepsilon\left(\alpha_{m}-\alpha_{m-1}\right)-(1+\varepsilon) \delta(\varepsilon / 2)^{m} \\
& \geq \varepsilon^{2}\left(\alpha_{m-1}-\alpha_{m-2}\right)-(1+\varepsilon) \delta \varepsilon^{m}\left(1 / 2^{m}+1 / 2^{m-1}\right) \\
& \geq \ldots \\
& \geq \varepsilon^{m}\left(\alpha_{1}-\alpha_{0}\right)-(1+\varepsilon) \delta \varepsilon^{m}\left(1 / 2^{m}+1 / 2^{m-1}+\ldots+1 / 2\right) \\
& \geq \varepsilon^{m}\left(\alpha_{1}-(1+\varepsilon) \delta\right) .
\end{aligned}
$$

Hence for $n>m$,

$$
\alpha_{n}-\alpha_{m}=\sum_{k=m}^{n-1} \alpha_{k+1}-\alpha_{k} \geq\left(\alpha_{1}-(1+\varepsilon) \delta\right) \sum_{k=m}^{n-1} \varepsilon^{k} .
$$

Thus

$$
\alpha_{n} \geq \alpha_{m}+\varepsilon^{m} \frac{1-\varepsilon^{n-m}}{1-\varepsilon}\left(\alpha_{1}-(1+\varepsilon) \delta\right)
$$

and (3) follows by letting $n \rightarrow \infty$.

4. Reflexivity and (P)-sequences. If $X$ is an infinite-dimensional reflexive Banach space, then according to $[\mathrm{R} 2]$ and $[\mathrm{Sh}], X$ contains a closed, convex and unbounded set with the AFPP. Shafrir [Sh] shows that if a Banach space $X$ contains a $(\mathrm{P})$-sequence $\left\{x_{n}\right\}$, then $C=\overline{\operatorname{conv}}\left\{n x_{n}: n \in \mathbb{N}\right\}$ has the AFPP. He also observes that a sequence $\left\{x_{n}\right\} \subset S_{X}$ which converges pointwise on $S_{X^{*}}$ to some $z \in X^{* *}$ which does not attain its norm is a (P)-sequence. According to a theorem of Odell and Rosenthal (see, e.g., [D, p. 236]), such sequences are always present in separable nonreflexive Banach 
spaces which do not contain an isomorphic copy of $\ell_{1}$. In this section we will show that a Banach space is not reflexive if and only if it contains a $(\mathrm{P})$ sequence. Consequently, all infinite-dimensional Banach spaces contain an unbounded closed convex set with the AFPP.

The following lemma strengthens one of the statements of Lemma 2.5 in the case of a separable Banach space.

LemMA 4.1. Suppose $X$ is a separable Banach space that contains no (P)-sequence. Let $\left\{x_{n}\right\}$ be a bounded sequence in $X$. Then $\left\{x_{n}\right\}$ contains a subsequence $\left\{x_{n_{k}}\right\}$ such that for every $x \in X$,

$$
\begin{aligned}
& \sup _{S_{X^{*}}}\left(x-\limsup _{k \rightarrow \infty} x_{n_{k}}\right)=\sup _{S_{X^{*}}}\left(x-\liminf _{k \rightarrow \infty} x_{n_{k}}\right), \\
& \inf _{S_{X^{*}}}\left(x-\limsup _{k \rightarrow \infty} x_{n_{k}}\right)=\inf _{S_{X^{*}}}\left(x-\liminf _{k \rightarrow \infty} x_{n_{k}}\right),
\end{aligned}
$$

and $\sup _{S_{X^{*}}}\left(x-\lim \sup _{k \rightarrow \infty} x_{n_{k}}\right)$ is attained.

Proof. First assume that $\left\{x_{n}\right\}$ contains a converging subsequence; using Lemma 3.1, choose a subsequence $\left\{x_{n_{k}}\right\}$ of this converging subsequence so that (5) is satisfied. Then $x-\lim \sup _{k \rightarrow \infty} x_{n_{k}}=x-\lim _{k \rightarrow \infty} x_{n_{k}}=y \in X$ and the supremum, that is, the norm of $y$, is attained.

Assume now that $\left\{x_{n}\right\}$ does not contain any converging subsequences. Define $S=S_{X^{*}}$ and consider $X$ as a subset of $\ell_{\infty}(S)$. Then $X$ is separable and $\left\{x_{n}\right\}$ is bounded. Choose a subsequence $\zeta=\left\{n_{k}\right\}$ of $\mathbb{N}$ so that (i) of Lemma 3.1 is satisfied. By (ii) of Lemma 3.1, both the equalities of (5) hold. Suppose there is some $x \in X$ such that

$$
\sup _{S_{X^{*}}}\left(x-\limsup _{k \rightarrow \infty} x_{n_{k}}\right) \text { is not attained. }
$$

Since $\left\{x_{n}\right\}$ is bounded, there is a subsequence $\xi$ of $\zeta$ such that $\lim \left\|x-x_{\xi}\right\|$ $=a$ exists. Since $\left\{x_{n}\right\}$ does not contain norm-convergent subsequences, $a>0$. Put $z_{n}=\left(x-x_{\xi(n)}\right) /\left\|x-x_{\xi(n)}\right\|$. We will show that $\left\{z_{n}\right\}$ is a $(\mathrm{P})$-sequence and this will be a contradiction. Since $\xi$ is a subsequence of $\zeta$, (i) of Lemma 3.1 continues to be satisfied when $\zeta$ is replaced with $\xi$. Hence by (ii) of the same lemma, we have

$$
\begin{aligned}
\sup _{f \in S_{X^{*}}} \limsup _{n \rightarrow \infty} f\left(z_{n}\right) & =a^{-1} \sup _{s \in S} \limsup _{n \rightarrow \infty}\left(x-x_{\xi(n)}\right)(s) \\
& =a^{-1} \sup _{s \in S} \liminf _{n \rightarrow \infty}\left(x-x_{\xi(n)}\right)(s) \\
& =\sup _{f \in S_{X^{*}}} \liminf _{n \rightarrow \infty} f\left(z_{n}\right) .
\end{aligned}
$$

We claim that $\sup _{f \in S_{X^{*}}} \lim \sup _{n \rightarrow \infty} f\left(z_{n}\right)$ is not attained. When combined with (7), this implies by Lemma 2.3 that $\left\{z_{n}\right\}$ is a $(\mathrm{P})$-sequence. Suppose that $\sup _{f \in S_{X^{*}}} \limsup _{n \rightarrow \infty} f\left(z_{n}\right)$ is attained. We will show that then also 
$\sup _{S_{X^{*}}}\left(x-\limsup _{k \rightarrow \infty} x_{n_{k}}\right)$ is attained and this will contradict (6). To this end, let $s \in S$ be such that

$$
\begin{aligned}
\sup _{f \in S_{X^{*}}} \limsup _{n \rightarrow \infty} f\left(z_{n}\right) & =a^{-1} \sup _{S} \limsup _{n \rightarrow \infty}\left(x-x_{\xi(n)}\right) \\
& =a^{-1} \limsup _{n \rightarrow \infty}\left(x-x_{\xi(n)}\right)(s) .
\end{aligned}
$$

Since $\xi$ is a subsequence of $\zeta$ and $\zeta$ satisfies (i) of Lemma 3.1, we have

$$
\begin{aligned}
\sup _{S} \limsup _{n \rightarrow \infty}\left(x-x_{\zeta(n)}\right) & =\sup _{s \in S} \limsup _{n \rightarrow \infty}\left(x-x_{\xi(n)}\right)=\limsup _{n \rightarrow \infty}\left(x-x_{\xi(n)}\right)(s) \\
& \leq \limsup _{n \rightarrow \infty}\left(x-x_{\zeta(n)}\right)(s) .
\end{aligned}
$$

Hence $\sup _{S} \lim \sup _{n \rightarrow \infty}\left(x-x_{\zeta(n)}\right)$ is indeed attained, contradicting (6).

Here is a variation of the above proof, perhaps slightly more efficient. Assume again that $\left\{x_{n}\right\}$ does not contain any converging subsequences. A simple diagonalization argument (see, e.g., [R1]) allows us to choose a subsequence $\eta$ of $\mathbb{N}$ so that for all $x \in X$ there exists $\lim _{n \rightarrow \infty}\left\|x-x_{\eta(n)}\right\|>0$. Next we choose a subsequence $\zeta=\left\{n_{k}\right\}$ of $\eta$ satisfying (i) of Lemma 3.1. By (ii) of Lemma 3.1, both equalities of (5) hold. If $\sup _{S_{X^{*}}}\left(x-\lim \sup _{k \rightarrow \infty} x_{n_{k}}\right)$ is not attained for some $x \in X$, then we can apply a similar argument to the one used above to show that $\left\{\left(x-x_{n_{k}}\right) /\left\|x-x_{n_{k}}\right\|\right\}$ is a $(\mathrm{P})$-sequence, and this again provides us with a contradiction.

The proof of the following proposition was inspired by the proof of the James theorem presented in [S].

Proposition 4.2. Let $X$ be a separable Banach space that contains no (P)-sequence. Then every bounded sequence $\left\{x_{n}\right\}$ in $X$ contains a subsequence which converges pointwise on $S_{X^{*}}$ to some $z \in X^{* *}$, and $z$ attains its norm on $S_{X^{*}}$.

Proof. Let $\left\{x_{n}\right\}$ be a bounded sequence in $X$. We may assume that $\left\{x_{n}\right\}$ is contained in the unit ball. Choose a subsequence $\left\{x_{n_{k}}\right\}$ of $\left\{x_{n}\right\}$ as described in Lemma 4.1 and put $z=\liminf _{k \rightarrow \infty} x_{n_{k}}$. We will show that $z(s)=\lim _{k \rightarrow \infty} x_{n_{k}}(s)$ for all $s \in X^{*}$. Then $z$ is linear and bounded on the unit ball of $X^{*}$, hence $z \in X^{* *}$. As $X$ contains no $(\mathrm{P})$-sequence, $\|z\|=$ $\sup _{f \in S_{X^{*}}} \lim _{k \rightarrow \infty} f\left(x_{n_{k}}\right)$ is attained by Lemma 2.5.

Define $S=S_{X^{*}}$ and consider $X$ as a subset of $\ell_{\infty}(S)$. Suppose to the contrary that there are some $s_{0} \in S$, a subsequence $\eta$ of $\left\{n_{k}\right\}$, and $b>0$ such that

$$
\left(x_{\eta(n)}-z\right)\left(s_{0}\right) \geq b>0 \quad \text { for all } n \in \mathbb{N} .
$$

Put $z_{n}=x_{\eta(n)}-z, A_{n}=\operatorname{conv}\left\{z_{i}: i \geq n\right\}$, and fix some $0<\delta, \varepsilon<1$ so that $(1+\varepsilon) \delta+2 \varepsilon<b$. We apply the construction of Lemma 3.2 to obtain 
$\left\{y_{n}\right\}$ and $\left\{\alpha_{n}\right\}$ as defined there. Then

$$
\begin{aligned}
y_{n}+z \in A_{n}+z & =\operatorname{conv}\left\{x_{\eta(i)}-z: i \geq n\right\}+z \\
& =\operatorname{conv}\left\{x_{\eta(i)}: i \geq n\right\} \subset X .
\end{aligned}
$$

Define $v=\lim \sup _{k \rightarrow \infty} x_{n_{k}}$. Since $\eta$ is a subsequence of $\left\{n_{k}\right\}$ and $y_{n} \in A_{n}=$ $\operatorname{conv}\left\{z_{i}: i \geq n\right\}$, we have

$$
\begin{aligned}
z & =\liminf _{k \rightarrow \infty} x_{n_{k}} \leq \liminf _{n \rightarrow \infty}\left(z_{n}+z\right) \leq \liminf _{n \rightarrow \infty}\left(y_{n}+z\right) \\
& \leq \limsup _{n \rightarrow \infty}\left(y_{n}+z\right) \leq \limsup _{n \rightarrow \infty}\left(z_{n}+z\right) \leq \limsup _{k \rightarrow \infty} x_{n_{k}}=v .
\end{aligned}
$$

By $(9), x=(1-\varepsilon) \sum_{k=1}^{\infty} \varepsilon^{k-1}\left(y_{k}+z\right) \in X$. Hence

$$
\sup _{S}\left((1-\varepsilon) \sum_{k=1}^{\infty} \varepsilon^{k-1} y_{k}\right)=\sup _{S}(x-z)=\sup _{S}(x-v) .
$$

The second equality above holds because $\left\{n_{k}\right\}$ has been chosen so that (5) be satisfied. By Lemma 4.1, there is some $s \in S$ such that

$$
\sup _{S}(x-v)=(x-v)(s) .
$$

For $m \in \mathbb{N}$, by (11) and by the definition of $\alpha_{n}$, we have

$$
\begin{aligned}
(1-\varepsilon) \lim \alpha_{n}= & \sup _{S}\left((1-\varepsilon) \sum_{k=1}^{\infty} \varepsilon^{k-1} y_{k}\right)=\sup _{S}(x-v) \\
= & {\left[(1-\varepsilon) \sum_{k=1}^{\infty} \varepsilon^{k-1}\left(y_{k}+z-v\right)\right](s) } \\
\leq & (1-\varepsilon) \sum_{k \neq m+1} \varepsilon^{k-1} y_{k}(s)+(1-\varepsilon) \varepsilon^{m}\left(y_{m+1}+z-v\right)(s) \\
\leq & (1-\varepsilon) \alpha_{m}+2(1-\varepsilon) \sum_{k>m+1} \varepsilon^{k-1} \\
& +(1-\varepsilon) \varepsilon^{m}\left(y_{m+1}+z-v\right)(s) .
\end{aligned}
$$

The first inequality above holds because $z-v \leq 0$ by (10). By (12) and Lemma 3.2,

$$
\begin{aligned}
\varepsilon^{m}\left(y_{m+1}+z-v\right)(s) & \geq \lim \alpha_{n}-\alpha_{m}-\frac{2 \varepsilon^{m+1}}{1-\varepsilon} \\
& \geq \alpha_{m}+\frac{\varepsilon^{m}}{1-\varepsilon}\left(\alpha_{1}-(1+\varepsilon) \delta\right)-\alpha_{m}-\frac{2 \varepsilon^{m+1}}{1-\varepsilon} .
\end{aligned}
$$

Hence for $m \in \mathbb{N}$,

$$
\left(y_{m}+z-v\right)(s) \geq\left(\alpha_{1}-(1+\varepsilon) \delta-2 \varepsilon\right) /(1-\varepsilon) .
$$


Since by (10), $\liminf _{m \rightarrow \infty} y_{m}+z-v \leq 0$, this means that $\alpha_{1} \leq(1+\varepsilon) \delta+2 \varepsilon$. However, since $y_{1}=\sum t_{i} z_{i}$ for some $t_{i} \geq 0, \sum t_{i}=1$, we also have

$$
\alpha_{1}=\sup _{S} y_{1} \geq \sum t_{i} z_{i}\left(s_{0}\right) \geq b>(1+\varepsilon) \delta+2 \varepsilon
$$

which is a contradiction.

Now we are ready to present the following characterization of reflexive Banach spaces.

Theorem 4.3. For a Banach space $X$ the following are equivalent:

(i) $X$ is reflexive;

(ii) every bounded sequence $\left\{x_{n}\right\}$ in $X$ contains a norm attaining subsequence, that is, a subsequence $\left\{x_{n_{k}}\right\}$ for which $\sup _{f \in S_{X^{*}}} \lim \sup f\left(x_{n_{k}}\right)$ is attained;

(iii) every bounded sequence $\left\{x_{n}\right\}$ in $X$ contains a subsequence $\left\{x_{n_{k}}\right\}$ for which $\sup _{f \in S_{X^{*}}} \liminf f\left(x_{n_{k}}\right)$ is attained;

(iv) $X$ does not contain any $(\mathrm{P})$-sequence.

Proof. (i) $\Rightarrow$ (ii). Let $\left\{x_{n}\right\}$ be a bounded sequence in a reflexive Banach space $X$. There is a subsequence $\left\{x_{n_{k}}\right\}$ of $\left\{x_{n}\right\}$ such that weak-lim $x_{n_{k}}=$ $x \in X$. Choose $f \in S_{X^{*}}$ so that $f(x)=\|x\|$. Then for any $h \in S_{X^{*}}$, we have $\lim h\left(x_{n_{k}}\right)=h(x) \leq\|x\|$.

(ii) $\Rightarrow$ (iii). Let $\left\{x_{n}\right\}$ be a bounded sequence in $X$. Choose a subsequence $\left\{x_{n_{k}}\right\}$ of $\left\{x_{n}\right\}$ and $h \in S_{X^{*}}$ so that

$$
\lim h\left(x_{n_{k}}\right)=\sup _{f \in S_{X^{*}}} \limsup f\left(x_{n_{k}}\right) .
$$

Then

$$
\begin{aligned}
\sup _{f \in S_{X^{*}}} \liminf f\left(x_{n_{k}}\right) & \geq \lim h\left(x_{n_{k}}\right)=\sup _{f \in S_{X^{*}}} \limsup f\left(x_{n_{k}}\right) \\
& \geq \sup _{f \in S_{X^{*}}} \lim \inf f\left(x_{n_{k}}\right) .
\end{aligned}
$$

Hence sup liminf $f\left(x_{n_{k}}\right)$ is attained at $h$.

(iii) $\Rightarrow$ (ii). By Lemma 3.1, there exists a subsequence $\zeta$ of $\mathbb{N}$ such that for every subsequence $\eta$ of $\zeta$,

$$
\sup _{f \in S_{X^{*}}} \limsup f\left(x_{\eta}\right)=\sup _{f \in S_{X^{*}}} \liminf f\left(x_{\eta}\right)
$$

Choose a subsequence $\eta$ of $\zeta$ and $h \in S_{X^{*}}$ so that there exists

$$
\lim h\left(x_{\eta}\right)=\sup _{f \in S_{X^{*}}} \liminf f\left(x_{\eta}\right) .
$$


Then

$$
\begin{aligned}
\sup _{f \in S_{X^{*}}} \limsup f\left(x_{\eta}\right) & \geq \lim h\left(x_{\eta}\right)=\sup _{f \in S_{X^{*}}} \liminf f\left(x_{\eta}\right) \\
& =\sup _{f \in S_{X^{*}}} \lim \sup f\left(x_{\eta}\right) .
\end{aligned}
$$

Hence sup $\lim \sup f\left(x_{\eta}\right)$ is attained at $h$.

(ii) $\Leftrightarrow$ (iv). This is proved in Lemma 2.5.

(iv) $\Rightarrow$ (i). Suppose $X$ does not contain any (P)-sequences and suppose for a contradiction that $X$ is not reflexive. Let $Y$ be a separable nonreflexive subspace of $X$. By Lemma 2.2, $Y$ also does not contain any $(\mathrm{P})$-sequences. This means that $Y$ contains an isomorphic copy of $\ell_{1}$ : if it did not, then according to $[\mathrm{Sh}], Y$ would contain a $(\mathrm{P})$-sequence. Let $\left\{x_{i}\right\}$ be an isomorphic $\ell_{1}$-basis in $Y$. By Proposition 4.2, it contains a subsequence which converges pointwise on $Y^{*}$. As this subsequence is again an $\ell_{1}$-basis, we may assume that $\left\{x_{i}\right\}$ already has this property. Let $T: \ell_{1} \hookrightarrow Y$ be the embedding for which $T\left(e_{i}\right)=x_{i}$; here $\left\{e_{i}\right\}$ is the usual basis of $\ell_{1}$. Then the dual mapping $T^{*}: Y^{*} \rightarrow \ell_{\infty}$ is surjective and we can choose $f \in Y^{*}$ so that $T^{*} f=$ $(-1,1,-1,1, \ldots)$. Then $f\left(x_{n}\right)=f\left(T e_{n}\right)=T^{*} f\left(e_{n}\right)$. Hence $f\left(x_{n}\right)=(-1)^{n}$, which is a contradiction. Consequently, $X$ is reflexive.

Corollary 4.4. Let $X$ be an infinite-dimensional Banach space. Then $X$ contains an unbounded closed convex set with the AFPP.

Proof. If $X$ is reflexive, then $X$ contains such a set according to [R2] and [Sh]. If $X$ is not reflexive, then it contains, by Theorem 4.3, a (P)-sequence $\left\{x_{n}\right\}$. By $[\mathrm{Sh}], C=\overline{\operatorname{conv}}\left\{n x_{n}: n \in \mathbb{N}\right\}$ has the AFPP.

\section{References}

[D] J. Diestel, Sequences and Series in Banach Spaces, Springer, Berlin, 1984.

[EK] R. Espínola and W. A. Kirk, Fixed points and approximate fixed points in product spaces, Taiwanese J. Math. 5 (2001), 405-416.

[GK] K. Goebel and W. A. Kirk, Classical theory of nonexpansive mappings, in: Handbook of Metric Fixed Point Theory, W. A. Kirk and B. Sims (eds.), Kluwer, Dordrecht, 2001, 49-91.

[GR] K. Goebel and S. Reich, Uniform Convexity, Hyperbolic Geometry, and Nonexpansive Mappings, Marcel Dekker, New York and Basel, 1984.

[J] R. C. James, Characterizations of reflexivity, Studia Math. 23 (1964), 205-216.

$[\mathrm{K}] \quad$ T. Kuczumow, A remark on the approximate fixed-point property, Abstr. Appl. Anal. 2003, no. 2, 93-99.

[P] J. D. Pryce, Weak compactness in locally convex spaces, Proc. Amer. Math. Soc. 17 (1966), 148-155.

[R1] S. Reich, Product formulas, nonlinear semigroups, and accretive operators, J. Funct. Anal. 36 (1980), 147-168.

[R2] - The almost fixed point property for nonexpansive mappings, Proc. Amer. Math. Soc. 88 (1983), 44-46. 
[Sch] E. Schechter, Handbook of Analysis and Its Foundations, Academic Press, San Diego, CA, 1997.

[Sh] I. Shafrir, The approximate fixed point property in Banach and hyperbolic spaces, Israel J. Math. 71 (1990), 211-223.

[Si] S. Simons, A convergence theorem with boundary, Pacific J. Math. 40 (1972), 703708.

[S] C. Stegall, Applications of Descriptive Topology in Functional Analysis, Univ. Linz, 1985.

Mathematical Institute

Czech Academy of Sciences

Žitná 25

CZ-11567 Praha, Czech Republic

E-mail:matouse@matsrv.math.cas.cz
Department of Mathematics The Technion - Israel Institute of Technology 32000 Haifa, Israel E-mail: sreich@tx.technion.ac.il

Received December 18, 2002

Revised version April 2, 2003 